LAWRENCE LIVERMORE NATIONAL LABORATORY

\title{
Spectroscopic Monitoring of Kidney Tissue Ischemic Injury
}

J. Fitzgerald, A. Michalopoulou,

C. Troppmann, S. G. Demos

\section{April 23, 2004}

SPIE conference Proceedings

Photonics West,

January 2004, San Jose, California, USA 
This document was prepared as an account of work sponsored by an agency of the United States Government. Neither the United States Government nor the University of California nor any of their employees, makes any warranty, express or implied, or assumes any legal liability or responsibility for the accuracy, completeness, or usefulness of any information, apparatus, product, or process disclosed, or represents that its use would not infringe privately owned rights. Reference herein to any specific commercial product, process, or service by trade name, trademark, manufacturer, or otherwise, does not necessarily constitute or imply its endorsement, recommendation, or favoring by the United States Government or the University of California. The views and opinions of authors expressed herein do not necessarily state or reflect those of the United States Government or the University of California, and shall not be used for advertising or product endorsement purposes.

This work was performed under the auspices of the U.S. Department of Energy by University of California, Lawrence Livermore National Laboratory under Contract W-7405-Eng-48. 


\title{
Spectroscopic monitoring of kidney tissue ischemic injury
}

\author{
Jason T. Fitzgerald, Andromachi P. Michalopoulou, Christoph Troppmann \\ Department of Surgery, University of California Davis, Medical Center, Stockton Blvd 2315, \\ Sacramento, California 95817 \\ Stavros G. Demos \\ Lawrence Livermore National Laboratory, 7000 East Avenue, Livermore, California 94551 \\ and Department of Urology, University of California Davis, Medical Center, Sacramento, CA
}

\begin{abstract}
Noninvasive evaluation of tissue viability of donor kidneys used for transplantation is an issue that current technology is not able to address. In this work, we explore optical spectroscopy for its potential to assess the degree of ischemic damage in kidney tissue. We hypothesized that ischemic damage to kidney tissue will give rise to changes in its optical properties which in turn may be used to asses the degree of tissue injury. The experimental results demonstrate that the autofluorescence intensity of the injured kidney is decreasing as a function of time exposed to ischemic injury. Changes were also observed in the NIR light scattering intensities most probably arising from changes due to injury and death of the tissue.
\end{abstract}

Keywords: Spectroscopy tissue diagnostic; Laser Spectroscopy; Tissue injury; Kidney

\section{INTRODUCTION}

Evaluation of the initial viability of donor tissues and organs used for transplantation is an important factor in augmenting the number of available organs by allowing for increased use of grafts from marginal donors and by predicting the success of the transplant operation. Faced with the current organ donor crisis in the United States, with demand far exceeding supply, the transplant community is increasingly attempting to use organs from potential donors previously not considered for donation, such as those with a significant previous medical history (for example hypertension, presence of vascular disease, donors older than 70 years of age). Other categories of marginal donors include those whose organs have been preserved due to logistical or procurement constraints for extended time periods, typically for greater than 30 hours in the case of kidneys. Because of the reluctance of many transplant centers to use these organs, many potentially transplantable, lifesaving grafts are currently discarded. Transplanted organs that prove to be nonviable clinically require a second operation in order to remove the nonfunctioning graft, and in the case of liver and heart transplants, immediate re-transplant in order to save the patient's life. Organ non-viability arises principally from pre-existing donor factors in combination with ischemia-reperfusion injury. Ischemia initiates injury by depriving cells of the energy needed to maintain ionic gradients and homeostasis. Reperfusion after transplantation exacerbates this damage by triggering an inflammatory reaction involving oxygen free radicals, endothelial factors and leukocytes [1]. This inflammatory reaction in turn leads to a significantly increased risk for rejection [2]. A reliable method for assessing graft viability at the time of transplantation would therefore allow avoiding transplanting organs that may not be viable to begin with and save the recipient considerable morbidity and mortality.

Transplanting organs with significant pretransplant warm ischemic damage may leave the recipient with unacceptably poor allograft function and adversely affect graft and patient survival. An important factor for assessing organ viability is how much warm ischemic damage has been sustained by a potentially transplantable organ. Tests of vascular resistance [3], enzymatic leakage from cells [4], electrolyte fluctuations and other ischemic changes [5] have been devised to answer this question, but unfortunately these tests are hindered by hypothermic preservation and by clinical practicality [6]. Currently, the only reliable way of assessing ischemic organ damage is to transplant it and monitor its function. 
Optical spectroscopy can measure changes in tissue by quantifying differences in a tissue's spectral properties - its ability to absorb, emit and scatter light at various wavelengths. Measurements can be made quickly and no manipulation of an organ, such as biopsy or attachment of perfusion tubing, required. Because no enzymatic assays are involved, optical spectroscopic measurements may not be hindered by hypothermic preservation methods. In the practice of clinical organ transplantation, the only practical time window for taking measurements of graft viability is during the relatively inert state of hypothermic preservation. However, no methods are available to reliably quantify warm ischemia occurring before transplantation in hypothermically preserved organs.

In this study, our aim was to determine whether optical spectroscopic methods employed during hypothermic preservation are capable of measuring the amount of warm ischemia sustained by a kidney before hypothermic preservation. We used two optical spectroscopic modalities - autofluorescence, which can quantify hypoxia-related tissue fluorophores, such as NADH [7-11], and light scattering, which can detect cellular and molecular tissue changes [12-16].

\section{EXPERIMENTAL ARRANGEMENT}

Adult male Wistar rats weighing 300-500g were anesthetized using $8-12 \mathrm{mg} / \mathrm{kg}$ sodium pentobarbital $(50 \mathrm{mg} / \mathrm{ml})$ injected intraperitoneally. Laparotomy was performed through a midline abdominal incision. 200 units of heparin sulfate were injected systemically via the inferior vena cava (IVC) to prevent thrombosis during renal artery and vein clamping. The left kidney and its vascular pedicle were then dissected free of surrounding fascia and fatty tissue, and the left renal vascular pedicle was clamped for 0, 5, 10, 20, 30, 60, 90 or 120 minutes. Eight rats were studied at each of these eight time points (total number of experimental animals: 64). In all cases, the right kidney was left undisturbed, as an internal control, until the conclusion of the ischemic period, at which time the aorta and IVC were clamped above and below the level of the renal arteries and veins. The left kidney was then unclamped and a venting incision was made in the juxtarenal IVC. $4 \mathrm{ml}$ of Euro-Collins electrolyte solution for kidney preservation (Baxter Healthcare Corp., Round Lake, IL) at $4^{\circ} \mathrm{C}$ were injected into the aorta just proximal to the renal arteries to flush the kidneys for hypothermic preservation. Bilateral nephrectomies were then performed and the kidneys stored in organ preservation solution and ice. Animals were sacrificed after nephrectomy.

Hypothermically preserved ischemic and control kidneys from each animal were placed adjacent to each other on ice. For each pair of kidneys, two spectroscopic methods - laser autofluorescence and polarized light scattering imaging were employed. The experimental setup is depicted in figure 1. An optical parametric oscillator (OPO) laser system equipped with a SHG module that is pumped at $355 \mathrm{~nm}$ by the third harmonic of a Nd:YAG laser was used to excite the pairs of normal (uninjured) and injured kidneys. The emitted light was used to obtain fluorescence images of the kidneys at different wavelengths selected by inserting the appropriate filters in front of the imaging system. Resulting autofluorescence images from each pair of kidneys were captured by a liquid nitrogen cooled CCD array (512 x 512 pixels). A camera lens was used to project the images to the CCD detector. The same imaging system was used to record cross-polarized light scattering images in the NIR spectral region. A white light source was employed with illumination delivered to the sample by a fiber optic bundle. At the output of the illumination fiber, the light was passed first through a near infrared (670 nm LP) filter and then through a linear polarizer. The net output provided polarized NIR illumination of the kidneys. A second polarizer with its orientation orthogonal with respect to the polarization orientation of the illumination was placed in front of the imaging system followed by a narrow band filter to select the imaging wavelength.

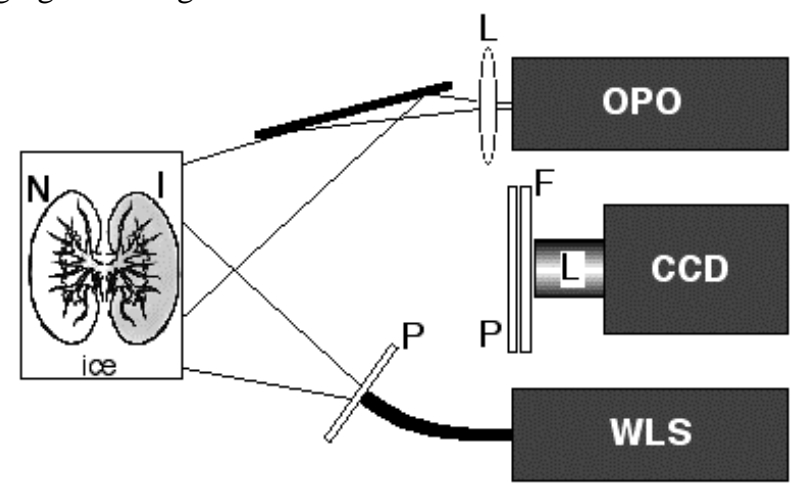

Figure 1: Schematic layout of the experimental set up. L: Lens, P: Polarizer, F: Filter, N: Normal and I: Injured kidneys, WLS: white light source. 
The autofluorescence images were obtained with the laser tuned at $355 \mathrm{~nm}$ to simultaneously excite with a nearly uniform illumination the surface of both kidneys. This laser illumination wavelength was obtained by second harmonic conversion of the tunable output of the OPO laser at $670 \mathrm{~nm}$. Excitation with this wavelength is known to penetrate tissue to a depth on the order of $100 \mu \mathrm{m}$. A set of filters was placed in front of the camera in order to record images at different wavelengths and spectral bands. These consisted of long pass (LP) filters at $395 \mathrm{~nm}$ and $725 \mathrm{~nm}$ and $20 \mathrm{~nm}$ bandwidth narrow band (NB) filters at $450 \mathrm{~nm}, 500 \mathrm{~nm}$ and $600 \mathrm{~nm}$.

In the cross polarized light scattering experiments, the back scattered light was passed through $700 \mathrm{~nm}$ or $800 \mathrm{~nm}$ filters (both with a bandwidth of $10 \mathrm{~nm}$ ) and images were captured by the CCD camera. We hypothetized that structural changes due to ischemia at the cellular or even molecular level can cause changes in the scattering and absorption coefficients, leading to differences in back scattering intensity. Images were analyzed using commercially available computer software to measure the ratio of autofluorescence or cross polarized light scattering image intensity of the ischemic to the control kidneys. The average image intensity from each kidney was estimated by calculating the average pixel intensity in the digitized images of the kidneys. Hypothermic preservation before image acquisition ranged between 30 and 180 minutes.

\section{EXPERIMENTAL RESULTS}

(a)

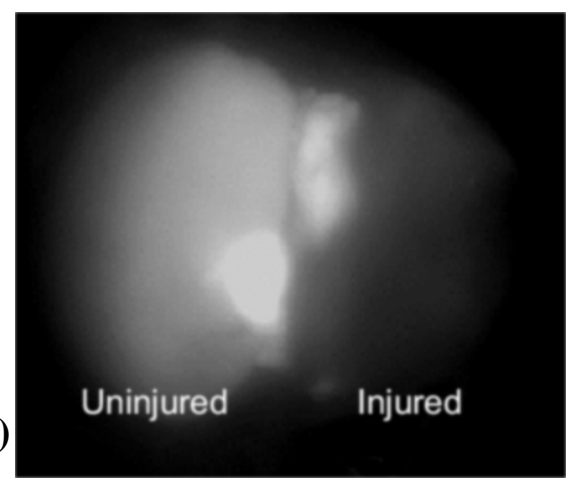

(b)

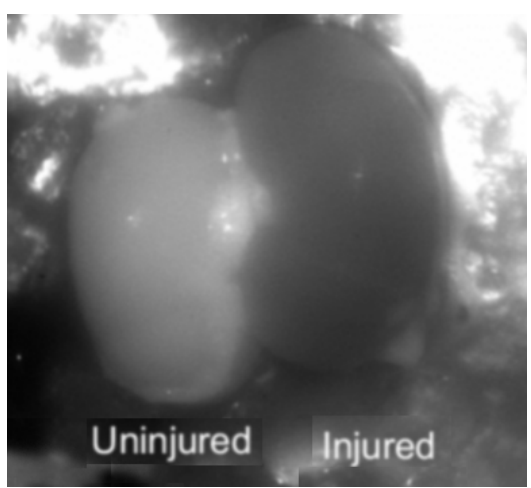

Figure 2: Images of the uninjured and injured kidneys from the same rat using a) Tissue autofluorescence at $450 \pm 20 \mathrm{~nm}$ under $335 \mathrm{~nm}$ excitation and b) cross polarized light scattering at $800 \mathrm{~nm}$.

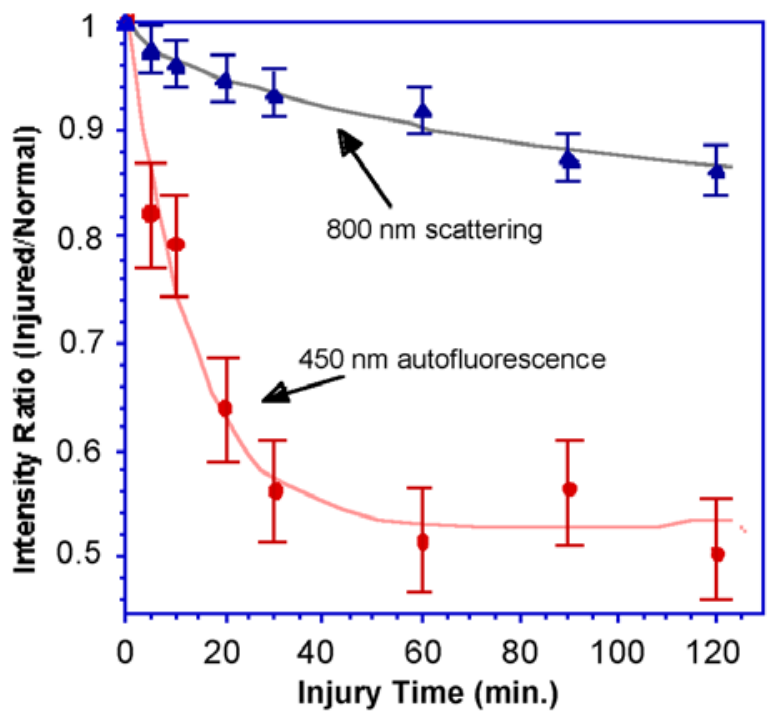

Figure 3: The injured/normal ratio of intensity of kidney images obtained using their autofluorescence at 450nm under $355 \mathrm{~nm}$ excitation ( solid circles) and cross polarized light scattering at $800 \mathrm{~nm}$ illumination (solid triangles). 
Figure 2a shows the autofluorescence image (at $450 \pm 20 \mathrm{~nm}$ ) under $335 \mathrm{~nm}$ excitation of a pair of kidneys where the right kidney was exposed to 120 minutes ischemic injury. Similarly, figure $2 \mathrm{~b}$ shows the NIR cross-polarized light scattering image (at $800 \pm 10 \mathrm{~nm}$ ) of a second pair of kidneys where the right kidney was exposed to 120 minutes ischemic injury. These images demonstrate the trend that we observed in our experimental data where the intensity of the autofluorescence as well as that of the light scattering images of the injured kidneys was lower than that of the corresponding normal kidney. This behavior is demonstrated in figure 3 where the normal/injured intensity ratios are plotted as a function of ischemia time using the autofluorescence images at $450 \pm 20 \mathrm{~nm}$ and the cross-polarized light scattering images at $800 \pm 10 \mathrm{~nm}$. The ratio of the normal/injured intensity obtained from the autofluorescence images of each pair of kidneys using the $395 \mathrm{LP}, 500 \mathrm{~nm} \mathrm{NB}, 600 \mathrm{~nm} \mathrm{NB}$ and $725 \mathrm{~nm}$ LP filters exhibited a similar profile to that obtained using the $450 \mathrm{~nm} \mathrm{NB}$ filter. This indicates that the changes of the ratio as a function of the ischemia time are due to reduction in the emission intensity of the ischemic kidney rather than due a change in the spectral profile. The spectral profiles of the emissions from the kidneys (control and injured) were also measured and confirmed that the overall intensity of the emission is reduced in the ischemic kidneys but the spectral characteristics of the emission remain almost unchanged.

\section{DISCUSSION}

The best overall differentiation in normal/injured intensity ratio with respect to ischemia time was achieved with autofluorescence images captured through the $450 \mathrm{~nm}$ filter (F-value 56.75, $\mathrm{p}<0.0001$, one-way ANOVA). Pairwise comparisons were significantly different between adjacent ischemia time points of 0 and 5 minutes, 10 and 20 minutes, and 30 and 60 minutes The difference between 5 and 10 minutes approached statistical significance $(\mathrm{p}=0.0728)$. The differences between most non-adjacent time points ( 5 and 20 minutes, for example) were significant. It must be noted that excitation in the $250-350 \mathrm{~nm}$ spectral range was tested and we found that $335 \mathrm{~nm}$ excitation offers the optimal contrast.

Using the light scattering technique, the best differentiation was achieved with images captured through the $800 \mathrm{~nm}$ filter (F-value 17.72, $\mathrm{p}<0.0001$, one-way ANOVA). Pairwise comparisons were significantly different between the adjacent ischemia time points of 20 and 30 minutes and 60 and 90 minutes. The differences between most non-adjacent time points (10 and 30 minutes, for example) were significant.

Between the $450 \mathrm{~nm}$ autofluorescence and the $800 \mathrm{~nm}$ light scattering methods, all adjacent and non-adjacent time points between 0 and 90 minutes were distinguishable. These two profiles were similar but not identical, indicating that the origins of tissue alterations due to ischemia probed by each method were different. A rational approach to quantifying the degree of unknown warm ischemia time would be to measure image intensity ratios between the injured kidney and the control kidney using both the $450 \mathrm{~nm}$ autofluorescence and the $800 \mathrm{~nm}$ light scattering methods and plotting the obtained values on standardized curves.

The advantage of using large area sampling provided by the autofluorescence and light scattering images, rather than the single point spectroscopic measurement, in this model is that the intensity from the entire kidney could be analyzed as a single measurement. Thus, meaningful results can be obtained despite heterogeneity in the degree of ischemia from point to point on the surface of the kidney. In contrast, spectral point measurements can analyze only a very small area of the kidney at one time. Because of the heterogeneity of injury across the kidney, data taken from one small point may not represent the whole surface.

The clinical aim of any measurement of pretransplant warm ischemia is to help in predicting a kidney graft's viability and function after transplantation. For the further development of optical spectroscopy as a pretransplant predictor of organ damage and posttransplant function, two critical steps remain apparent. First, while the measurements taken in this study correlated with the degree of warm ischemia time, it would be important clinically to correlate with actual pathophysiological measurements of tissue damage, such as capillary leak or creatinine clearance. Given the degree of variability in biological systems and the different factors that influence how tissue will respond to ischemia for a specific duration of time, optical spectroscopic measurements may correlate even more closely with physiological ischemic damage than with ischemia time. Second, and most importantly, such measurements must be correlated with posttransplant renal function. Ideally, this would lead to an absolute threshold measurement distinguishing a viable from a non-viable kidney. 


\section{ACKNOWLEDGMENT}

This work was performed in part at Lawrence Livermore National Laboratory under the auspices of the U.S. Department of Energy under Contract W-7405-Eng-48. This research is supported by the Lawrence Livermore National

Laboratory and the Center for Biophotonics, an NSF Science and Technology Center managed by the University of California, Davis, under Cooperative Agreement No. PHY 0120999.

\section{REFERENCES}

1. Torres, J, Cruzado, JM, Grinyo, JM. Ischemia and Reperfusion Injury in Transplantation, Transplantation Proceedings, 31, 2217-2218 (1999).

2. Halloran, PF, Melk, A, Barth, C. Rethinking Chronic Allograft Nephropathy: The Concept of Accelerated Senescence, J Am Soc Nephrol 10:167-181 (1999)

3. Inman S, Osgood R, Plott W, Richardson B, Lewis R. Identification of kidneys subjected to pre-retrieval warm ischemic injury during hypothermic perfusion preservation. Transplant Proc 1998; 30 (5): 2309.

4. Kievit JK, Nederstigt AP, Oomen AP, Janssen MA, Schoot L, Kootstra G. Release of alpha-glutathione Stransferase (alpha GST) and pi-glutathione S-transferase (pi GST) from ischemic damaged kidneys into the machine perfusate--relevance to viability assessment. Transplant Proc 1997; 29 (8): 3591.

5. Kootstra G, Kievit JK, Heineman E. The non heart-beating donor. Br Med Bull 1997; 53 (4): 844.

6. Stubenitsky BM, Booster MH, Nederstigt AP, Kievit JK, Jacobs RW, Kootstra G. Kidney preservation in the next millenium. Transpl Int 1999; 12 (2): 83.

7. Chance B, Williams GR. Respiratory enzymes in oxidative phosphorylation. I. Kinetics of oxygen utilization. J Biol Chem 1955; 217 (1): 383.

8. Barlow $\mathrm{CH}$, Harken AH, Chance B. Evaluation of cardiac ischemia by NADH fluroescence photography. Ann Surg 1977; 186 (6): 737.

9. Shehada R, Marmarelis, V., Mansour, H., Grundfest, W. Laser induced fluorescence attenuation spectroscopy: detection of hypoxia. IEEE Transactions on Biomedical Engineering 2000; 47 (3): 301.

10. Barlow C, Rorvik, D., Kelly, J. Imaging epicardial oxygen. Annals of Biomedical Engineering 1998; 26 : 76.

11. Mayevsky A, Nakache R, Merhav H, Luger-Hamer M, Sonn J. Real time monitoring of intraoperative allograft vitality.Transplant Proc 2000; 32 (4): 684.

12. Ba AM, Guiou M, Pouratian N, et al. Multiwavelength optical intrinsic signal imaging of cortical spreading depression. J Neurophysiol 2002; 88 (5): 2726.

13. Tao L, Masri D, Hrabetova S, Nicholson C. Light scattering in rat neocortical slices differs during spreading depression and ischemia. Brain Res 2002; 952 (2): 290.

14. Mendelson Y, Cheung PW, Neuman MR, Fleming DG, Cahn SD. Spectrophotometric investigation of pulsatile blood flow for transcutaneous reflectance oximetry. Adv Exp Med Biol 1983; 159: 93.

15. Anderson NM, Sekelj P. Light-absorbing and scattering properties of nonhaemolysed blood. Phys Med Biol 1967; 12 (2): 173.

16. Schmitt JM, Mihm FG, Meindl JD. New methods for whole blood oximetry. Ann Biomed Eng $1986 ; 14$ (1): 35. 